which corresponds to the time of the fluorescent event. This suggests that the defect center fluorescence is a unique, nonrandom event compared with the background signal. Zero correlation in this case also implies that only a single defect center is responsible for the event.

The overall collection efficiency in this experiment is still very poor (0.0014). The researchers suggest that one improvement is to "couple an emitting dipole to a microcavity so it will emit light in a single mode." They also said that better collection optics and samples will also improve efficiency. The researchers hold that despite low collection efficiency with the current setup, the relative simplicity of this experiment provides a good platform for future development.

JUNE LAU

\section{Quantum Mechanical Bond Breaking Characteristics Explain Fracture Anisotropy of Silicon}

In the June 5 issue of Physical Review Letters, scientists from the Max Planck Institut für Metallforschung in Stuttgart and the Universidad Autonoma de

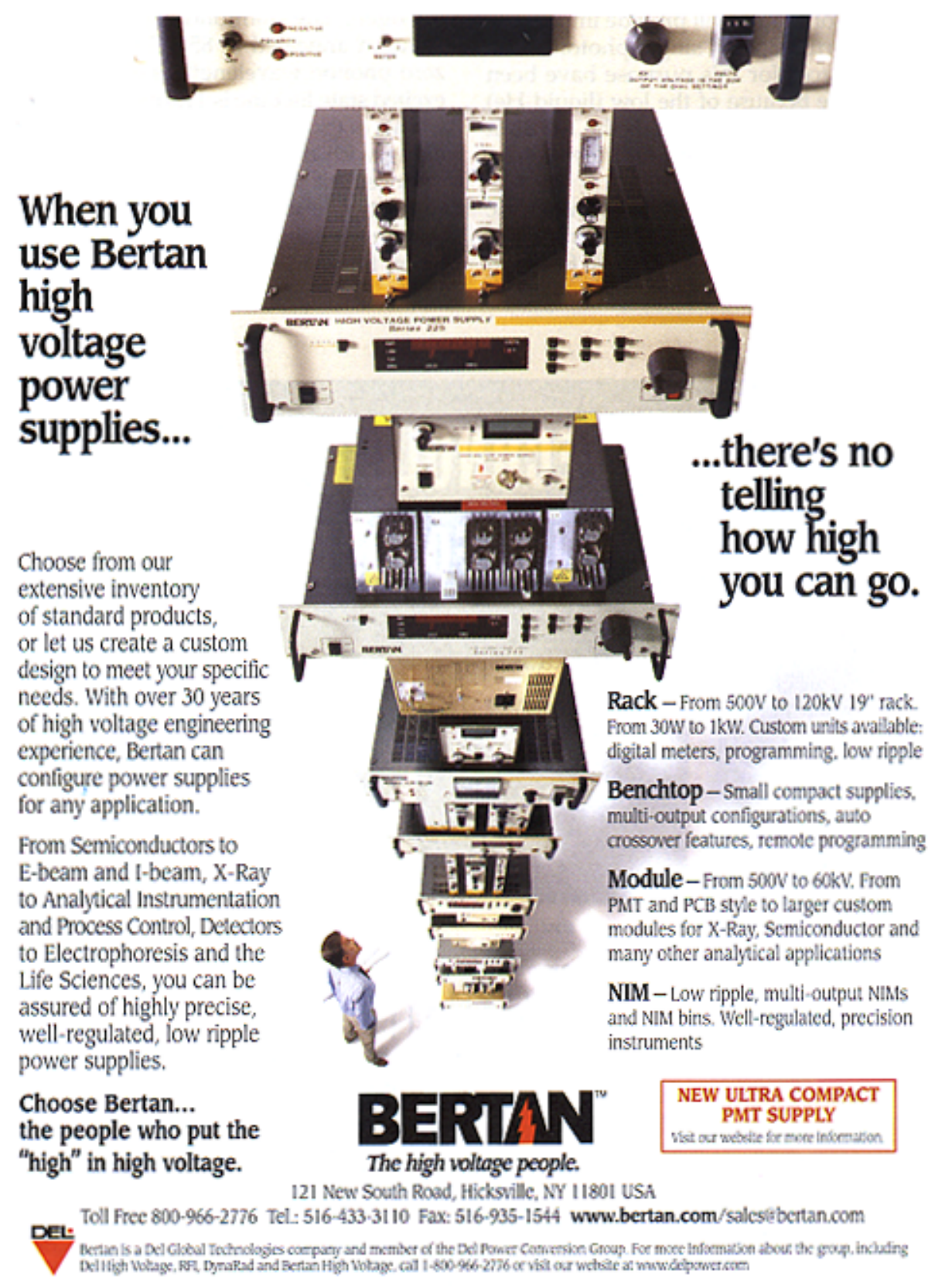

Circle No. 12 on Inside Back Cover
Madrid have reported quantum mechanical simulations of the bond breaking process at the tip of a crack in silicon which explain the well-documented cleavage anisotropy of this material.

Cracks in silicon propagate on two types of cleavage planes and clearly prefer one particular direction on both of these planes. This experimental finding cannot be explained with arguments based on the Griffith criterion, which relates fracture resistance to the surface energy of the fracture surfaces. A more thorough theoretical analysis is difficult because cracks form on the atomic scale but extend to macroscopic dimensions. However, the enormous increase in computing power recently has now opened new opportunities for such studies.

In the present case, the atoms around the crack tip were first loaded with the stress field of a macroscopic crack. The energy and all the forces on the atoms were then calculated $a b$ initio using density functional theory. Upon increasing the externally applied load, the bond breaking process and the relaxations of the surrounding atoms were then monitored. Scientists Rubén Pérez and Peter Gumbsch said that the surprising result of the simulations was that the bond breaking at the crack tip proceeds differently for the different crack orientations. While the crack tip bonds smoothly lengthened for the easy propagation direction, the bond length remained almost unchanged for the difficult orientation until the external load reached a critical value at which the bond abruptly broke like a snapping elastic spring.

The discontinuous bond breaking is preceded by a significant load sharing between several bonds at the crack tip, which effectively shields the crack tip bond from the applied load. This leads to a socalled trapping of the crack, which stabilizes it up to loads far above the Griffith value. Consequently, the macroscopically observed fracture anisotropy can be directly viewed as a result of the difference in the way the atomic bonds break.

\section{D Photonic Bandgap Structure Significantly Improves Performance of Laser Emission from a Conjugated Polymer System}

Having recently demonstrated the feasibility of surface-emitting conjugated polymer lasers using a one-dimensional feedback mechanism, researchers in Germany now report the development of a mechanically flexible polymer laser utilizing two-dimensional distributed feedback (2D-DFB) due to a two-dimensional photonic bandgap structure. Investigators from the Ludwig-Maximilians University 
in Munich, the Fraunhofer Institute for Solar Energy Systems in Freiburg, and The Max Planck Institute for Polymer Research in Mainz say that this advance significantly reduces the lasing threshold and improves the efficiency of organic laser devices. These results were announced in the September 11 issue of Applied Physics Letters.

A mechanically flexible poly(ethylene terephthalate) (PET) substrate with an acrylic coating was embossed with a $2 \mathrm{D}$ periodic height modulation nanopattern using ultraviolet (UV) radiation. The active laser material, a ladder-type poly(p-phenylene) (LPPP), was then deposited in a 300-nm thick layer on this substrate by spincoating. A Ti:sapphire laser producing pulses of approximately $150 \mathrm{fs}$ duration at a wavelength of $400 \mathrm{~nm}$ was used for excitation. When focused to a spot approximately $150 \mu \mathrm{m}$ in diameter on the LPPP surface, nearly diffraction limited monomode laser emission was observed perpendicular to the surface above a pump pulse energy of $1.2 \mathrm{~nJ}$. The 2D photonic bandgap structure significantly limits lasing from other lateral modes. The peak intensity of the emission occurred at $491 \mathrm{~nm}$. Comparison of the 2D laser with a one-dimensional (1D) periodic height modulated laser of the same maximum amplitude showed a dramatic increase in the emission intensity in the $2 \mathrm{D}$ case, as well as a $30 \%$ reduction in the laser threshold energy. Also, the divergence of emission was drastically reduced in the $2 \mathrm{D}$ laser, resulting in highly directed nonpolarized emission.

The researchers explain the observed effects in terms of the Laue formulation

\section{SBIR Update}

Diversified Technologies, Inc. (Bedford, Massachusetts) has been awarded $\$ 3,000,000$ under a total of six Phase I and Phase II Small Business Innovation Research (SBIR) grants from the Department of Energy to extend the firm's PowerMod $^{\mathrm{TM}}$ solid-state high power electronics technology to higher voltages and currents, and achieve faster switching times.

Advanced Refractory Technologies, Inc. (Buffalo, New York) has been awarded a $\$ 750,000$ Phase II SBIR contract from the U.S. Marine Corps for the development and implementation of a lightweight/ durable aluminum metal matrix composite track system for the Advanced Amphibious Assault Vehicle. for the feedback mechanism in a 2D photonic band structure, involving elastic Bragg scattering to couple wavevectors having the same energy.

TIM PALUCKA

\section{Bottom-Gate Geometry Increases Materials Options in All-Polymer Integrated Circuits}

Investigators at the Philips Research Laboratories in The Netherlands have shown that bottom-gate structures for organic field-effect transistors open the door to a wide variety of polymeric materials, including the high mobility semiconducting materials pentacene and regioregular poly(3-hexylthiophene), or P3HT. Reporting in the September 4 issue of Applied Physics Letters, G.H. Gelinck,
T.C.T. Geuns, and D.M. de Leeuw also demonstrated that low-ohmic vertical interconnects (vias) between the top and bottom layer can be etched photochemically, making eventual scaling up of the technology feasible.

Top-gate structures sandwich the semiconductor between the first electrode and the gate dielectric, imposing materials compatibility restraints on the organic semiconductor employed. Previous work using a top-gate electrode with polythienylenevinylene (PTV) as the semiconductor resulted in transistors with low field-effect mobilities (approximately $1 \times 10^{-3} \mathrm{~cm}^{2} / \mathrm{Vs}$ ). Since the semiconductor is not sandwiched in the bottom-gate structure, materials compatibility is a lesser concern; high mobility organic

\section{The future of the multibillion dollar display industry will be determined by research results in phosphor science and technology. You can see the opportunities at: \\ Over 70 Technical Papers selected by a distinguished International Program Committee • Poster Sessions • Single-track Conference for Enhanced Interaction \\ The Sixth International Conference on the Science and Technology of Display Phosphors}

\section{November 6 - 8 Catamaran Resort Hotel San Diego, California}

\section{Topics}

Plasma Phosphors

Organic EL Materials \& Devices

Inorganic EL Materials \& Devices

FED Phosphors

- Synthesis

- Properties

- Degradation \& Screening

\section{Speakers from the United}

States, Japan, Germany,

Belgium, U.K., Ukraine,

Finland, Korea, Canada,

France, Mexico, Russia, The Netherlands, \& PR China.

\section{Many opportunities for discussion}

Sponsored by:

The Phosphor Technology Center of Excellence

The U.S. Defense Advanced Research Projects Agency

The Society for Information Display
For registration information contact: Mark Goldfarb Palisados Institute for Research Services 411 Lafayette St., 2nd FI. New York, NY 10003 Phone $212460-8090 \times 202$ Fax $212460-5460$ URL: http://wrww,sid.org/conf/conf,html
Circle No. 16 on Inside Back Cover 